\title{
PLASMA ACTIVATED SYNTHESIS OF MACROMOLECULAR COMPOUNDS
}

\author{
V. Chernyak ${ }^{a, *}$, V. Iukhymenko ${ }^{a}, \mathrm{~K}_{\text {. Iukhymenko }}{ }^{a}, \mathrm{O}_{\text {. Prysiazhna }}{ }^{a}$, \\ D. HAmAZin ${ }^{a}$, E. OBeremoK ${ }^{a}$, V. KYslenko ${ }^{a}$, A. Liptuga ${ }^{b}$ \\ ${ }^{a}$ Faculty of Radio Physics, Electronics and Computer Systems, Taras Shevchenko National University of Kyiv, \\ Volodymyrska Street 64/13, 01601, Kyiv, Ukraine \\ ${ }^{b}$ V. Lashkaryov Institute of Semiconductor Physics, pr. Nauky 41, 03028, Kyiv, Ukraine \\ * chernyak_v@ukr.net
}

\begin{abstract}
The paper is devoted to study of the plasma-activated synthesis of organic compounds with optically activity. Synthesis was carried out in plasma-liquid system with a rotating gliding discharge submerged in a liquid. The initial reagents of synthesis were ethanol, ammonia, and $\mathrm{CO}_{2}$. The possibility of the influence of the electric field direction on the optical activity of the products of plasma-activated synthesis is shown.
\end{abstract}

Keywords: plasma, synthesis, chirality, plasma-liquid system, $\mathrm{CO}_{2}$, polarimetry.

\section{Introduction}

Throughout the fifty-year history of the intensive development of plasma chemistry, one of the main areas of its research has been the synthesis of both organic and inorganic substances. It has been shown that the use of isothermal plasma in combination with rapid quenching of the plasma composition when the temperature changing at a rate of $10^{7}-10^{8} \mathrm{~K} \mathrm{~s}^{-1}$ and non-equilibrium conditions can be effective in the synthesis. The plasma-activated synthesis has made it possible to obtain new unknown conventional chemistry nanomaterials such as fullerenes and nanotubes $[1,2]$.

But as in conventional chemistry, the problem of chiral synthesis of nonchiral substances remains unsolved. The solution to this problem can have significant implications for plasma applications (agroplasma, plasma medicine, pharmacology, the food industry, etc.) $[3,4]$.

Chirality is the geometric property of a spatial structure to be incompatible with its mirror image in a perfect flat mirror. Chiral molecules can exist as two isomers (enantiomers), which are mirror images of each other. Feature of chemical transformations with the participation of chiral molecules is the manifestation of chirality only when interacting with other chiral molecules. That is why the chemical synthesis of chiral isomers from non-chiral starting reagents results only in a racemic mixture of chiral isomers (50\% right $/ 50 \%$ left). A possible cause of the racemate mixture may be the quasi-equilibrium nature of the chemical processes and, accordingly, provided that all directions of the translational and rotational motion of the molecules of the reagents are equal.

Little-known dynamic plasma-chemical systems based on rotational gliding discharge (RGD) [5] may be promising for chiral synthesis. Since, such systems allow to realize both translational and rotational mo- tion in a certain direction due to gas dynamics and to control the direction of motion of charged particles in ionic molecular processes by an electric field. A fundamental feature of RGD is the perpendicularity of the electric field of the axis of rotation of the gas flow [6].

Another property of chiral molecules as physical objects is the optical activity in their interaction with polarized light. The left isomer rotates the light polarization plane to the left, the right isomer turns right at exactly the same angle.

This work is devoted to the study of optical activity (rotation of the polarization plane of light by matter) of organic synthesis in a plasma-liquid system with a rotating gliding discharge submerged in a liquid.

\section{Experimental Setup and Methods}

Experimental setup with a rotating gliding discharge submerged in liquid is shown in Figure 1. The plasma generator consists of a central electrode (anode), the upper flange (cathode) and dielectric chamber with holes for tangential supply of working gas. The central part of the anode has a conical shape with a hole in the centre. The diameter of the hole is $3 \mathrm{~mm}$. Rotating gliding discharge burns between the cathode and anode. The distance between cathode and anode is $1 \mathrm{~mm}$. The quartz tube filled with tested liquid is placed on the cathode.

The polarity of the electrodes in the system could be changed. When the central electrode was anode, the reactor (the part in contact with the liquid and the flange) was a cathode. When the central electrode was a cathode, the reactor (the part in contact with the liquid and the flange) was the anode. A mixture of ethanol and ammonia was used as the test liquid. Gas was supplied tangentially to the axis of the system. $\mathrm{CO}_{2}$ was used as the working gas. The $\mathrm{CO}_{2}$ flow was 


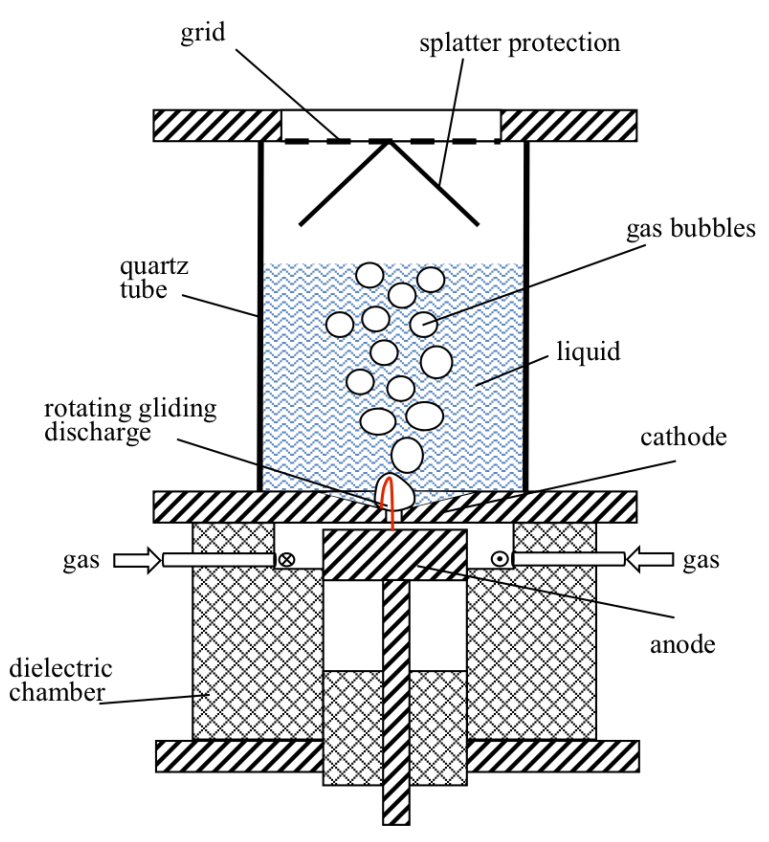

Figure 1. Experimental setup with rotating gliding discharge submerged in liquid.

$101 \mathrm{~min}^{-1}$. There was a mesh above the liquid with a cone to catch the splashes of liquid.

A mixture of ethanol $\left(\mathrm{C}_{2} \mathrm{H}_{5} \mathrm{OH} 96 \%\right)$ and ammonia $\left(\mathrm{NH}_{4} \mathrm{OH} 25 \%\right)$ was treated. The volume of each substance was $50 \mathrm{ml}$. $\mathrm{CO}_{2}$ was used as the working gas. Processing time was $10 \mathrm{~min}$. The discharge current was $100 \mathrm{~mA}$, the discharge voltage varied from 1.15 to $3 \mathrm{kV}$ depending on the polarity of the electrodes (when the central electrode was anode - the voltage was $1.15-1.5 \mathrm{kV}$, when the central electrode was cathode - the voltage was $3 \mathrm{kV}$ ). The direction of $\mathrm{CO}_{2}$ supply was varied: in one case, the gas supply was counterclockwise (viewed from above on the electrodes), in the other case the gas supply occurred clockwise (if viewed from above on the electrodes). The volume of the processed solution was $100 \mathrm{ml}$.

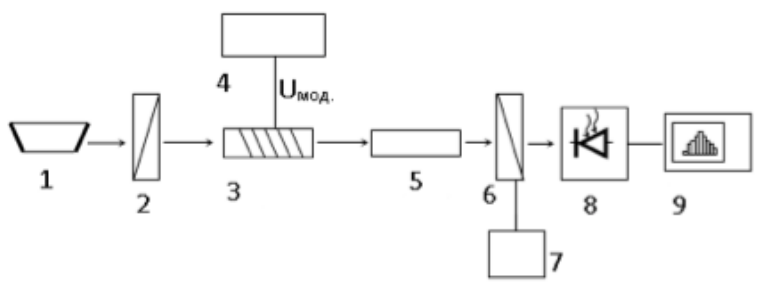

Figure 2. The scheme of laser modulation polarimeter. 1 - helium-neon laser $(\lambda=0.63 \mu \mathrm{m}) ; 2$ - fixed polarizer; 3 - Faraday modulator; 4 - modulating $U_{\text {mod }}$ generator; 5 - cuvette with the test sample; 6 - the analyzer; 7 - a nuance of a reference optical microscope with a limb; 8 - photodetector; 9 - digital spectrum analyzer.

After processing, the solutions were analyzed using a polarimeter. A $2 \mathrm{~cm}$ cuvette was used. A laser modulation polarimeter was used to study of optical activity of the samples. The scheme a polarimeter is shown in Figure 2.

The use of a digital spectrum analyzer and a nonlimiting optical microscope with a limb allowed us to determine the optical activity of the samples with an error not exceeding 10 angular seconds.

Absorption spectra of the treated liquids were measured to analyze the composition of the liquid. Liquid and solid phase compositions were also studied using IR transmission on an «Infralum FT-801» device (Figure 3).

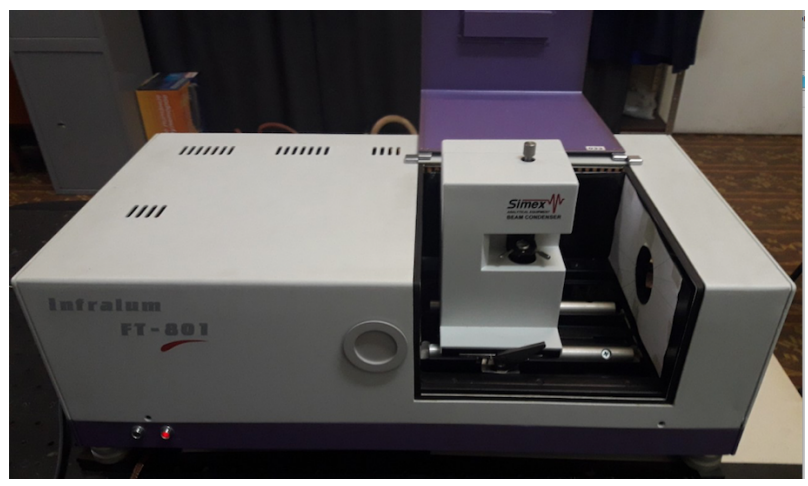

Figure 3. Infralum FT-801.

\section{Results and Discussion}

During the processing of solutions, a solid phase was formed in the system. Over time (within a week) it went into the liquid phase. This occurred both during the treatment of the solution with plasma (purge $\mathrm{CO}_{2}$ with the discharge-on) and with the purge with non-activated plasma $\mathrm{CO}_{2}$ (discharge-off). A typical photo of the plasma solution treatment is presented in Figure 4.

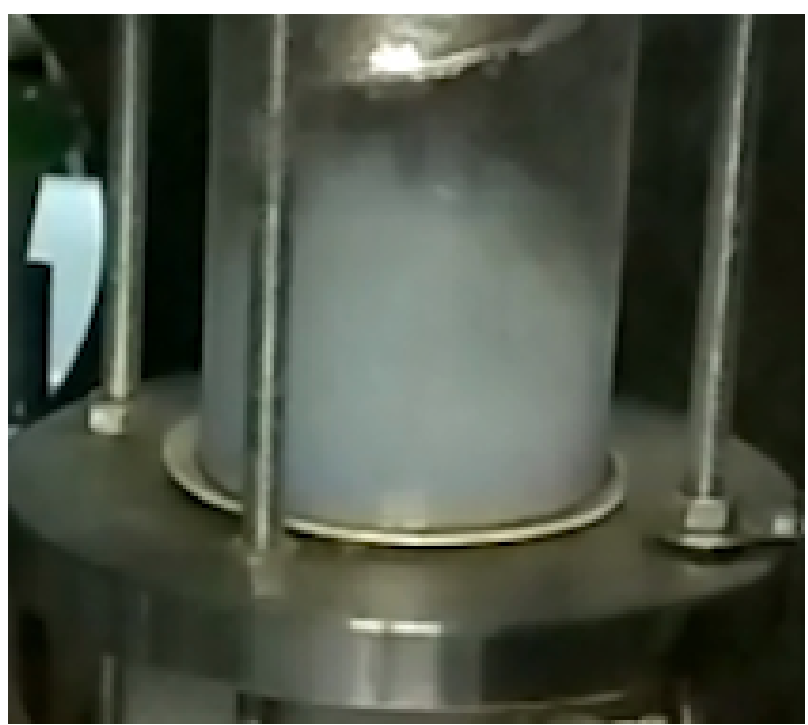

Figure 4. Photo of the system when treating $\mathrm{C}_{2} \mathrm{H}_{5} \mathrm{OH}$ $(96 \%)+\mathrm{NH}_{4} \mathrm{OH}(25 \%)$ solution with $\mathrm{CO}_{2}$.

After analysis of the treated solutions on the polarimeter, the heterogeneity of the indicators over 
time were observed. For the operating modes of the system in the presence of a discharge (for different polarity of the electrodes and the direction of supply of the plasma-forming gas), the effect of the deviation of rotation angle of the plane of light polarization is

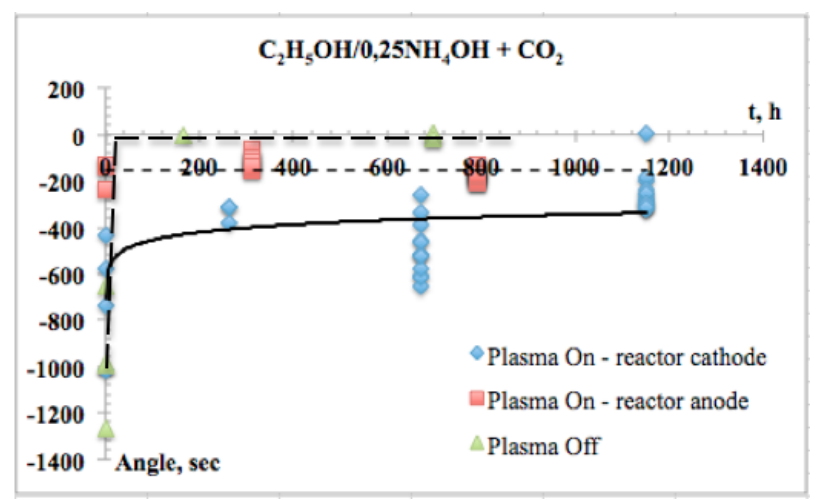

Figure 5. Optical activity of solutions after treatment in a plasma-liquid system under different modes of operation.

much greater than the measurement error, but has the same orientation for all modes (clockwise). The deviation angle is up to 10 angular minutes clockwise. After 1100 hours the effect persists, but the deviation decreases and is up to 5 angular minutes (Figure 5). For quite a long time, the optical activity of the solutions is changing. This may be due to the fact that chemical transformations continue in solutions for a long time.

When the solutions are treated in a plasma-off mode but only supplied with gas, a large deviation of the angle is observed on the first day after treatment (2050 minutes after treatment). However, the next day there is no deviation of the angle or within the margin of error. It should also be noted that the deviation angle depends on the composition of the solutions being treated. If you reduce the volume of ammonia in the solution by half, the deviation angle will be several minutes. If you reduce the ethanol volume in the solution by half, the deviation angle will be within the error.

The results of composition analysis of solutions before and after treatment in the system obtained by IR transmission are presented in Figure 6 and 7 .

From the analysis of the spectra it can be seen that in spectra of solution (ethanol $(50 \mathrm{ml})+$ ammonia $(50 \mathrm{ml}))$ which was treated in a plasma-liquid system with a rotating gliding discharge, submerged in liquid, there are components of water, ethanol and ammonia are present. However, unlike all the original components of the mixture, an additional peak appears in the treated solution around $1500-1600 \mathrm{~cm}^{-1}$. In the initial solution this band is not present (Figure 7). The intensity of this band is greater when the solution is treated in the presence of plasma.

In Figure 8 presents the absorption spectra of solutions after treatment in the system. The spectra were

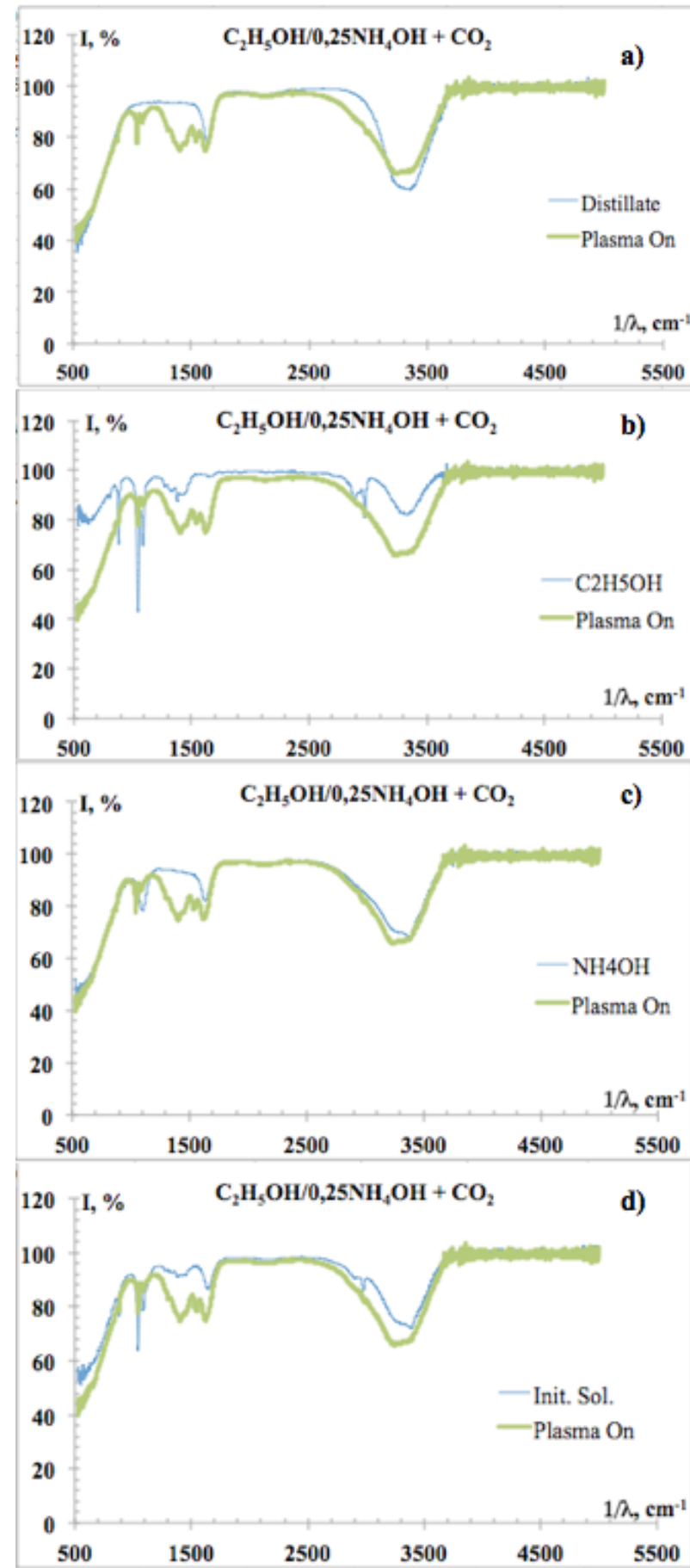

Figure 6. IR transmission spectra of distillate (a), ethanol (b), ammonia (c), initial solution (d) and treated solution (Plasma On - reactor cathode).

measured immediately after treatment.

As it can be seen, the absorption spectra of the treated solutions when plasma-on and when plasmaoff are different from the initial solution. For plasmatreated solutions, the absorption spectra have a small peaks in the area 420-480 $\mathrm{nm}$.

During quite a long time after processing in the system (more than 80 days) this tendency persists (Figure 9). This behaviour of the spectra is preserved when repeating the experiments. 


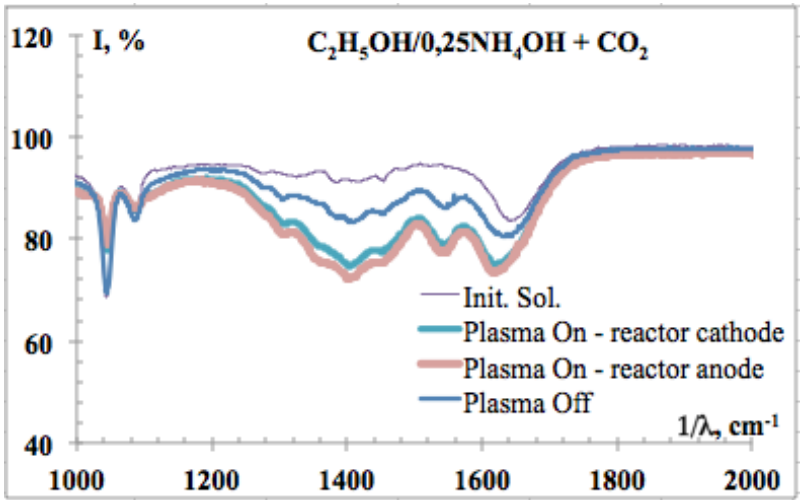

Figure 7. IR transmission spectra of initial solution and solutions treated in the presence of plasma at different polarities of the electrodes and without plasma.

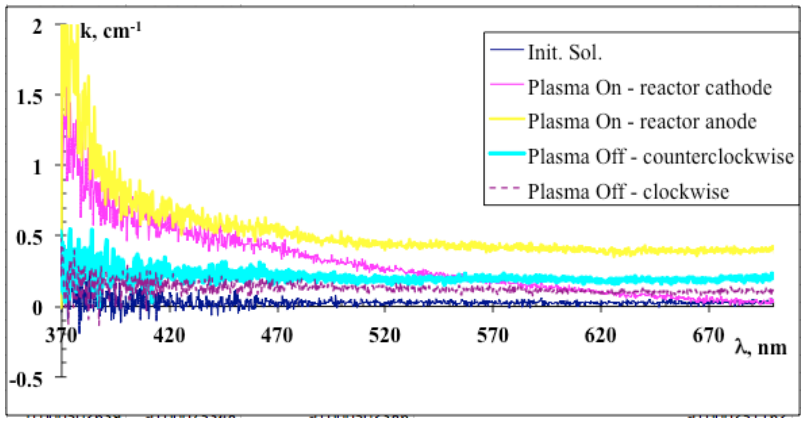

Figure 8. Absorption spectra of the initial solution and solutions treated in a plasma-liquid system with rotating gliding discharge in the presence of plasma and without.

\section{Conclusions}

1. The possibility of plasma-chemical synthesis of an optically active substance when all the starting reagents are not active in the synthesis is shown.

2. It is shown that the plasma activation of synthesis may lead to the production of optically active substances, the level of activity, which persists for more than 1000 hours, that is in principle not possible in purely chemical synthesis.

3. In the plasma-off mode the effect of changing of the polarization angle disappears for the next day.

4. In the plasma-on mode reduction of the ethanol volume in the solution in half does not change the polarization angle.

5. The possibility of the influence of the electric field direction on the optical activity of the products of plasma-activated synthesis is shown.

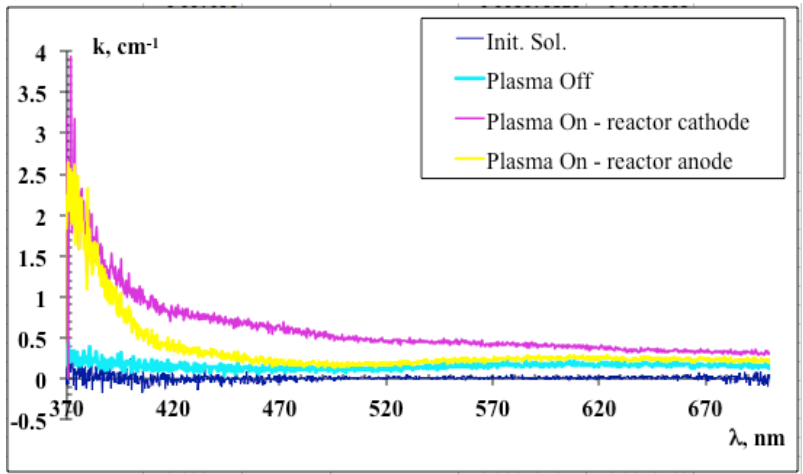

Figure 9. Absorption spectra of initial solution and solutions treated in a plasma-liquid system with rotating gliding discharge in the presence of plasma and without. 80 days after treatment.

\section{References}

[1] A. Fridman. Plasma chemistry. Cambridge university press, 2008.

[2] R. Snoeckx, A. Rabinovich, D. Dobrynin, A. Bogaerts, and A. Fridman. Plasma-based liquefaction of methane: The road from hydrogen production to direct methane liquefaction. Plasma Processes and Polymers, 14(6):1600115, 2017. doi:10.1002/ppap. 201600115.

[3] https://himija-online.ru/ organicheskaya-ximiya/aminokisloty/alanin.html.

[4] I. Leenson. Chirality in living and non-living nature, 2016.

[5] V. Chernyak. Gas discharge plasma in dynamics system as a noneqilibrium plasma sources. In Proc. 3rd Czech-Russian Seminar on Electrophysical and Thermophysical Processes in Low-temperature Plasma, Brno, Nov. 16-19,, pages 94-99, 1999.

[6] V. Chernyak, V. Iukhymenko, K. Iukhymenko, M. Klochok, O. Kolomiiets, D. Hamazin, and D. Chernolutsky. Features of atmospheric pressure discharges with a transverse component of the velocity of gas flow to the current channel. Problems of Atomic Science and Technology. Series: Plasma Physics, 118(6):185-188, 2018. 